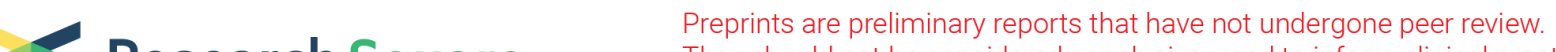 $\begin{array}{ll}\text { Research Square } & \text { They should not be considered conclusive, used to inform clinical practice, } \\ \text { or referenced by the media as validated information. }\end{array}$
}

\section{Comparative Profiling of Small RNAs Originated From Two Varieties of Chinese Cabbage (Brassica Rapa) With Distinct Leaf Traits}

\section{XU XIN LIU}

Shanghai Institute of Plant Physiology and Ecology: Institute of Plant Physiology and Ecology Shanghai Institutes for Biological Sciences https://orcid.org/0000-0002-3464-6438

\section{XIANG YU}

Shanghai Institute of Plant Physiology and Ecology: Institute of Plant Physiology and Ecology Shanghai Institutes for Biological Sciences

\section{HAN WANG}

Shanghai Institute of Plant Physiology and Ecology: Institute of Plant Physiology and Ecology Shanghai Institutes for Biological Sciences

YU KE HE ( $\nabla$ ykhe@sibs.ac.cn )

Shanghai Institute of Plant Physiology and Ecology: Institute of Plant Physiology and Ecology Shanghai Institutes for Biological Sciences

\section{Research Article}

Keywords: Brassica rapa, miRNA, comparative profiling, leaf variance, small RNA deep sequencings

Posted Date: April 8th, 2021

DOl: https://doi.org/10.21203/rs.3.rs-398360/v1

License: (c) (1) This work is licensed under a Creative Commons Attribution 4.0 International License. Read Full License

Version of Record: A version of this preprint was published at Genetic Resources and Crop Evolution on August 10th, 2021. See the published version at https://doi.org/10.1007/s10722-021-01236-y. 


\section{Abstract}

The species Brassica rapa includes many important vegetable crops. For a genome-wide survey of small RNAs in B. rapa, we performed massively parallel small RNA deep sequencing by using two representative varieties of non-heading Chinese cabbage (B. rapa ssp. chinensis) that are distinct in leaf shape, size, color and curvature. In total, 13.30 million small RNA reads were generated from Huaq, a variety with up-curved blades and wide petioles, and 14.69 million for Wut, a variety with down-curved blades and narrow petioles. After normalization, about half of small RNA reads in each variety were mapped to the published reference genome of B. rapa. In Huaq seedlings, unique small RNAs were much more than in Wut seedlings. Among them, 45 miRNAs were up-regulated or down-regulated in one variety, compared with those in another variety. Numbers of ta-siRNAs (trans-acting siRNAs) and ra-siRNAs (repeat-associated siRNAs) in Huaq seedlings were more than those detected in Wut seedlings, while gene-derived siRNAs (siRNAs derived from the sense and antisense strands of annotated genes regeion) in Huaq seedlings were less than in Wut seedlings. Especially, bra-miR156, bra-miR166 and bra-miR319 and one of ta-siRNAs that play important role in leaf shape, leaf size, leaf curvature and phase transition were differentially expressed between two varieties. In addition, total number of small RNAs derived from chloroplast genome of Wut was more than that of Huaq. The differentially-expressed small RNAs on genome-wide level provides the clue for the study of small-RNA-mediated morphology of leaves and is useful for developing of small RNA markers for leaf traits desirable for high yield and quality.

\section{Introduction}

Brassic rapa (B. rapa) includes many kinds of important vegetable crops. The edible parts of these crops are leafy heads, leaf clusters, modified blades, and modified petioles. The distinct morphologies exhibited by subspecies of $B$. rapa represent one of the most spectacular evolutionary changes that illustrate the structural evolution of plants under domestication. There are multiple genetic and epigenetic variances between different genotypes in B. rapa and A. thaliana, both of which diverged from a common ancestor separated approximately 13-17 million years ago (Mun et al., 2009). The B. rapa genome contains triplicated homologous counterparts of corresponding segments comparing to the genome of Arabidopsis. thaliana due to the events of entire genome triplication (Wang et al., 2011b).

Small RNAs of Chinese cabbage are involved in heat responses(Wang et al., 2011a; Yu et al., 2011). It is not known how small RNAs contributes to diversity of leaf shape during evolution for different genotypes. In general, small RNAs cause transcriptional gene silencing by guiding heterochromatin formation at homologous loci, whereas others lead to posttranscriptional gene silencing through mRNA degradation or translational inhibition. Some of small RNAs are derived from and target foreign nucleic acids such as viruses and transgenes, but most of them are derived from endogenous loci and regulate a multitude of developmental and physiological processes, and response to abiotic or biotic stress (Chen, 2009).

Small RNAs is a big family, and there are four major classes of endogenous small RNAs in plants: microRNAs(miRNA), transacting siRNAs(ta-siRNA), natural antisense transcript-derived siRNA(nat-siRNA) 
and heterochromatic siRNAs (hc-siRNA). Small RNAs can arise through distinct biogenesis routes and function through loading into Argonaute-protein-containing effector complexes (Jamalkandi and Masoudi-Nejad, 2009). miRNA are produced from loci which encoded a fairly long transcript(pri-miRNA), which is processed by DICERLIKE1(DCL1), HYPONASTIC LEAVES 1(HYL1) and SERRATE(SE) to a shorter hairpin structure, the pre-miRNA. The mature miRNA is recruited by AGO1 into the RNAi silencing complex(RISC). RNAase activity of AGO1 is directed to the target of the miRNA (Voinnet, 2009). ta-siRNAs are a second class of siRNA produced under direction of miRNA. Like miRNAs, ta-siRNAs are taken up by AG01 into a silencing complex. miRNA are found in plants and animals but ta-siRNA have so far been identified only in plants (Montgomery et al., 2008).

Small RNAs have been implicated as factors in many aspects of plant development, which comprise several new layers of gene regulation, affecting transcription, stability, localization and translation. For examples, miR156 controls vegetative phase and floral transition (Wang et al., 2008; Wu et al., 2006); miR160 and miR167 controls hormone biosynthesis and signaling (Chuck et al., 2007; Mallory et al., 2005; Reyes and Chua, 2007); miR165/166 monitors leaf polarity and morphogenesis (Juarez et al., 2004; Sieber et al., 2007); miR164a is required for regulation of CUC gene expression in the developing leaf lamina (Nikovics et al., 2006); miR398 has the roles in stress resistance such as drought (Liu et al., 2008), over-oxidation (Sunkar et al., 2006), and phosphate starvation (Fujii et al., 2005).

Leaf develops from the shoot apical meristem (SAM), a group of stem cells at the apex of the shoot. On the flanks of the SAM a group of cells loses indeterminacy and becomes a leaf primordium. These cells take cues from their position in the SAM to begin to divide, initially outwards to form a peg (a group of cell of leaf primordium), and then divisions become localized to the margin between the upper and lower side to form the leaf blade or lamina (Steeves, 1989). Many factors can affect the development of leaf from leaf primordia to a mature leaf with adaxial side and abaxial side (Chitwood et al., 2009; Juarez et al., 2004; Nagasaki et al., 2007; Wenkel et al., 2007).

Huaq and Wut are two varieties of non-heading Chinese cabbage. They are distinct in shape, size, color and curvature of leaves although the shortened shoots and roots are similar. The yield and quality of Chinese cabbage are largely dependent on shape, size, color and curvature of leaves. Recently, several microRNAs (miRNAs) and siRNAs have been identified to regulate leaf shape, curvature and size in Arabidopsis since they are involved in leaf polarity, cell division, auxin response and phase transition (Liu et al.; Wu et al., 2007; Yu et al., 2005). A question remains whether and how small RNAs regulate leaf phenotypes of Chinese cabbage, a closely relatives of Arabidopsis. At now, Small RNA deep sequencing is widely used for genomics research and is an important tool for analysis on the functional complexity of transcriptome (Hollister et al., 2011). It provides an approach to understand why close relative species or varieties present diverse phenotypes, and types and expression levels of some small RNAs could be regarded as molecular markers for leaf traits as well. Identification of differently expression of small RNAs between Huaq and Wut offers the opportunity to understand the molecular mechanisms involved in leaf variance at transcriptional and post-transcriptional level. 


\section{Materials And Methods}

Plant material and small RNA sequencing

Huaq-1 and Wut-1 are the two inbred lines of Huaq (B. rapa. ssp. chinensis var. huaq) and Wut (B. rapa. ssp. chinensis var. wut). All plants were grown under a $16 / 8 \mathrm{~h}$ light/dark photoperiod at $22^{\circ} \mathrm{C}$ for 3 weeks, the above-ground portions of the seedlings were harvested, quick-frozen immediately with liquid nitrogen and stored at $-80^{\circ} \mathrm{C}$. Total RNAs were extracted using TRIzol Reagent (Invitrogen). RNA concentration was measured by Eppendorf Biophotometer 6121. DNA was removed by digestion using TURBO DNA-free kit (Ambion). RNA samples were sent to Keygene N.V., Wageningen in Netherlands where the samples were prepared by using the MIRVana MIRNA Isolation Kit (Ambion, Austin, TX, USA) and sequenced on Illumina GAll sequencer, according to the Alternative v1.5 Protocol (Illumina, San Diego, CA, USA).

Alignments of raw reads with reference genome

Recently, genome sequence of Chinese cabbage (Chiifu-401-42) genotype has been reported (Wang et al., 2011b). This genome sequence has made us possible to match small RNA sequences on the good reference data. After removing adaptors, the small RNA with length of $17 \sim 36$ nucleotides (nt) were mapped to genomic sequences (BRAD) ${ }^{1}$ of Chinese cabbage (B. rapa ssp. pekinensis. cv Chiifu-401-42) by SOAP2 (short oligonucleotide alignment program) (Li et al., 2009) with the 0 or 1 mismatch. For learning small RNAs derived from chloroplast, mitochondria, rRNAs, tRNA, TE (transposable elements) and snoRNAs (small nucleolar RNA), all reads were aligned with Arabidopsis TAIR9 cDNA database.

Annotation of the origin of small RNAs

Small RNAs which are miRNAs were identified by comparing to the former annotated miRNA of B. rapa (Yu et al., 2011). The ta-siRNAs that are homologous to Arabidopsis homologs were aligned to the TAS genes in B. rapa. Small RNAs that were generated from the genes were selected by comparing the location of small RNAs with Brassica gene (http://brassicadb.org). Then, these genes were annotated on the basis of homologous genes of Arabidopsis and Gene Ontology (GO) analysis. Small RNAs that have more than 50 multiple locations on genome sequences were considered as ra-siRNA (repeat-associated siRNA). Meanwhile, small RNAs were aligned to Arabidopsis chloroplast genome, mitochondria genome, transposable elements, ribosome RNA, tRNA and snoRNA.

Analysis of expressional difference

The abundance of all mapped reads was normalized to the number of reads per 10 million (RP10M). Small RNAs which only detected in one cultivar with more than 10 reads was considered cultivarenriched. The identical reads whose P-value cutoffs of 0.01 and 2 -fold change of Huaq-to-Wut ratio were considered to be different in expression levels, and defined as Huaq-predominant, and vice versa. The Pvalue was calculated based on the Bayes inference: $\mathrm{P}=0.5^{(\mathrm{N} 1+\mathrm{N} 2)} /\left([\mathrm{N} 1 /(\mathrm{N} 1+\mathrm{N} 2)]^{\mathrm{N} 1} \times[\mathrm{N} 2 /(\mathrm{N} 1+\mathrm{N} 2)]^{\mathrm{N} 2}\right)$ 
where $\mathrm{N} 1$ and $\mathrm{N} 2$ are the reads of two samples, respectively. The small RNAs that were specifically expressed in one variety rather than another were defined as specific reads.

\section{Results}

The distinct phenotypes of Huaq and Wut seedlings

Huaq-1 and Wut-1 are two inbred lines of Huaq and Wut, respectively. To demonstrate difference in leaf shape and leaf architecture between two varieties, we carefully observed leaf shape, size, color and curvature of the two inbred lines at rosette stage (6-week old). For the variety Huaq, leaves were big; blade was oval in shape; and petioles were extremely wide and thick (Fig. 1A, B, Table 1). For the variety Wut, leaves were small; blades were round and dark-green; and petioles were long and narrow. Especially, Huaq petioles were characterized with inward curvature (Fig. 1C, D).

Table 1. Leaf parameters of Huaq and Wut. The data were recorded 6 weeks after sowing and were the mean of 20 individuals.

\begin{tabular}{|lll|}
\hline Leaf traits & Huaq & Wut \\
\hline Leaf length $(\mathrm{cm})$ & $21.5 \pm 1.2$ & $10.1 \pm 0.9$ \\
\hline Leaf width $(\mathrm{cm})$ & $10.1 \pm 0.7$ & $5.3 \pm 0.4$ \\
\hline Blade length $(\mathrm{cm})$ & $14.3 \pm 1.1$ & $4.6 \pm 0.2$ \\
\hline Blade width $(\mathrm{cm})$ & $10.2 \pm 0.5$ & $5.3 \pm 0.3$ \\
\hline Blade shape index $(\mathrm{cm})$ & $2.1 \pm 0.1$ & $0.9 \pm 0.1$ \\
\hline Leaf area $\left(\mathrm{cm}{ }^{2}\right.$ per leaf) & $47.6 \pm 3.7$ & $22.7 \pm 1.8$ \\
\hline Petiole width $(\mathrm{cm})$ & $7.1 \pm 0.5$ & $1.2 \pm 0.1$ \\
\hline Petiole length $(\mathrm{cm})$ & $7.2 \pm 0.4$ & $6.6 \pm 0.4$ \\
\hline Petiole thickness $(\mathrm{cm}$ & $0.9 \pm 0.1$ & $0.4 \pm 0$ \\
\hline
\end{tabular}

To show the difference in leaf curvature between Huaq and Wut, we performed quantitative measurement of length and width of blade and petiole. Blade shape index of Huaq was much higher than that of Wut (Table 1). Blade area of Huaq was more than two times, compared with that of Wut. The width and thickness of Huaq petioles were higher than those of Wut petioles. The leaf parameters confirmed that the two varieties were distinct in shape and size of blade and petioles.

Characterization and classification of small RNA reads

Before analysis, all small RNA reads were filtered out with low-complexity, low quality and repetitive reads. In total, 13,295,284 reads for Huaq and 14,690,798 reads for Wut were obtained ${ }^{2}$. For convenient 
comparison of abundance between two small RNA libraries, the raw reads of Huaq and Wut were normalized to the number of reads per 10 million (RP10M) (Table 2). For mapping to the genome, the sequence length of reads shorter than $17 \mathrm{nt}$ were filtered out. The qualified reads of small RNAs were finally aligned with the Chinese cabbage reference genome (http://brassicadb.org) by using the SOAP2 software, set one mismatch as threshold ${ }^{2}$. After analysis, $63.07 \%$ of Huaq and $61.88 \%$ of Wut within the raw reads were mapped to $B$. rapa genome (Wang et al., 2011b) (Table 2).

\section{Table 2. Classification of small RNAs of unique reads and total reads in non-heading Chinese cabbage that mapped to the reference genome of B. rapa. "shared" indicates common small RNAs between Huaq and Wut.}

\begin{tabular}{|llll|}
\hline Types of small RNAs & \multicolumn{3}{l|}{ Unique reads } \\
\hline & Huaq & Wut & Shared \\
\hline Total small RNA & 2109930 & 1737905 & 497645 \\
\hline Gene-derived small RNAs & 441632 & 419485 & 116385 \\
\hline miRNAs & 75 & 75 & 72 \\
\hline ta-siRNAs & 88 & 81 & 40 \\
\hline ra-siRNAs & 45727 & 40912 & 21674 \\
\hline Other & 1622408 & 1277352 & 359474 \\
\hline
\end{tabular}

\begin{tabular}{|lllll|}
\hline \multicolumn{4}{l}{ Total reads } & \\
& Huaq & Wut & Shared in Huaq & Shared in Wut \\
\hline Total small RNA & 6307078 & 6187666 & 4110151 & 4702562 \\
\hline Gene region derived small RNAs & 1420374 & 1503518 & 960410 & 1159442 \\
\hline miRNAs & 452657 & 307479 & 452652 & 307313 \\
\hline ta-siRNAs & 434 & 307 & 371 & 264 \\
\hline ra-siRNAs & 234632 & 184809 & 200495 & 157679 \\
\hline Other & 4198981 & 4191553 & 2496223 & 3077864 \\
\hline
\end{tabular}

As a result, 6.30 million and 6.18 million small RNA reads in Huaq and Wut were mapped on B. rapa reference genome, meanings that Huaq plants produce more small RNAs than Wut plants. Among them, there were about 2.10 million unique reads for Huaq, and 1.73 million for Wut. Interestingly, 1.42 million reads were matched on sense or antisense strands of annotated genes in Huaq, while 1.50 million reads on the genes in Wut. Between two varieties, total numbers of miRNAs, ta-siRNAs, and ra-siRNAs were 
different (Table 2). Huaq and Wut shared 497,645 unique reads, indicating the distinct difference on accumulation of common small RNAs between Huaq and Wut.

In total, 75 miRNA families were detected in Huaq and Wut ${ }^{2}$. However, 0.45 million miRNA reads were derived from Huaq and 0.30 million from Wut, revealing that more miRNAs were generated from Huaq than Wut. More than 80 putative ta-siRNAs were generated from TAS3 precursor in both varieties. Additionally, about 20000 small RNAs belonged to ra-siRNAs.

The expression of a number of small RNAs was different between Huaq and Wut. 7725 reads were Huaqspecific since they were not detected in Wut; and 4767 reads were Wut-specific since they were not detected in Huaq (Table 3). Meanwhile, 15805 small RNAs were Huaq-predominant $(P<0.01)$ since they were more abundant in Huaq than in Wut; and 18048 small RNAs were Wut-predominant since they were more abundant in Wut than in Huaq. In addition, numbers of gene-derived small RNAs and ra-siRNAs were significantly different.

Table 3. Classification of different expressional small RNAs in non-heading Chinese cabbage. "specific" indictates the small RNAs specifically expressed in one variety. "predominant" means that the abundance of the small RNAs in one variety are two times more than in another variety. "Gene region derived" indicates the small RNAs matched on sense or antisense strand of the annotated coding genes.

\begin{tabular}{|lllll|}
\hline & $\begin{array}{l}\text { Huaq- } \\
\text { specific }\end{array}$ & $\begin{array}{l}\text { Wut- } \\
\text { specific }\end{array}$ & $\begin{array}{l}\text { Huaq- } \\
\text { predominant }\end{array}$ & $\begin{array}{l}\text { Wut- } \\
\text { predominant }\end{array}$ \\
\hline Total small RNA & 7725 & 4767 & 15805 & 18048 \\
\hline $\begin{array}{l}\text { Gene region derived small } \\
\text { RNAs }\end{array}$ & 229 & 107 & 1590 & 593 \\
\hline miRNAs & 0 & 1 & 34 & 10 \\
\hline ta-siRNAs & 0 & 0 & 1 & 0 \\
\hline ra-siRNAs & 22 & 107 & 925 & 246 \\
\hline
\end{tabular}

Although the total numbers of unique reads were approximately the same between Huaq and Wut, the length of small RNA reads were substantially different (Fig. 2). The peaks of length for unique reads were the same at $24 \mathrm{nt}$ in both varieties. However, the abundance of small RNAs with $21 \sim 24 \mathrm{nt}$ in Huaq was more than that of Wut. In contrast, the abundance of $25 \sim 32$ nt small RNAs in Wut was less than that of Huaq.

Many small RNA reads were perfectly matched with chloroplast genome. They were designated as chloroplast-related small RNAs. Among them, some were matched with nuclear sequences as well, and thus filtered out; and the rest were chloroplast-specific small RNAs (csRNA). The situation was the same for mitochondria-specific small RNAs (mitRNA). To detect the proportion of csRNA and mitRNA, small 
RNA reads were aligned to Arabidopsis Tair 9 chloroplast genome, mitochondria genome, and cDNA database (Fig. 3). The result showed that proportion of csRNAs in Wut seedlings was more than in Huaq seedlings in terms of number of unique reads and abundance of reads.

Small RNA distribution in chromosome

We calculated the distribution of small RNA reads in chromosome scale at 100-kilobase pair $(100 \mathrm{~kb})$ windows along ten chromosomes (Fig. 4). There were many hot spots on genome where small RNA reads are enriched. Interestingly, the peaks of the total small RNA abundance between Huaq and Wut were distributed similarly. In contrast, the hot sites of small RNA transcription between Arabidopsis species were very diverse, especially between two most related species Arabidopsis lytrata and Arabidopsis thaliana (Hollister et al., 2011). One exception was that at the end of chromosome 9th of Wut, there was a hot spot.

Differential expression of individual miRNAs between Huaq and Wut

Recently, many species of higher plants have been identified to have species-specific miRNAs (Axtell and Bowman, 2008). To verify whether some miRNAs existed in one variety but not in another, we compared the sequences of miRNAs in Huaq and Wut seedlings. Among 78 miRNAs, 3 miRNAs were Wut-specific (Supplementary Table 1). They were bra-miR403, bra-miR5711, bra-miR399f.

Comparison of miRNA expression between Huaq and Wut showed that 76.9\% miRNAs were expressed predominantly in Huaq seedlings, and $23.1 \%$ miRNAs predominant in Wut seedlings. We classified the miRNAs into 5 groups according to the range of abundance $(>10000,1001-10000,251-1000,100-250$, $<100$ reads) (Fig. 5). bra-miR156a was more abundant in Wut seedlings than in Huaq seedlings, while bra-miR159a and bra-miR166a accumulated more in Huaq seedlings than in Wut seedlings. miR156 is the most ancient miRNAs and has been found in almost all land plant lineages (Axtell and Bowman, 2008). bra-miR1885b.3 and bra-miRNA319a were upregulated 2.5 folds, compared with those of Wut seedlings. Meanwhile, bra-miRNA169b, bra-miR5711, bra-miR5725 and bra-miR395a were up-regulated in Wut seedlings, compared with those of Huaq seedlings.

ta-siRNA in Huaq and Wut seedlings

Alignment of small RNAs with ta-siRNAs of Arabidopsis indicated that only ta-siRNAs from B. rapa TAS3 (BrTAS3) transcripts were presented in Huaq and Wut datasets. TAS3 ta-siRNAs are generated from miR390-mediated cleavage, and its target genes are two auxin response factor genes, ARF3 and ARF4, which promote abaxial identity and expression of adult traits in leaves (Adenot et al., 2006). We choose BrTAS3 precursors as reference to align all small RNAs. More than 80 small RNAs were matched with TAS3, but only one of them was conserved with TAS3a D7(+) (5'-TTCTTGACCTTGTAAGACCCC-3'), the known ta-siRNA in Arabidopsis. This ta-siRNA was located on the 5th chromosome of the genome of B. rapa. It was not known whether the other small RNAs derived from TAS3 precursors acted as ta-siRNAs in B. rapa. 
Small RNAs aligned to the annotated genes of B. rapa

For analysis of function of small RNAs, we aligned small RNA reads with the annotated genes of $B$. rapa. More than 0.44 million small RNAs in Huaq and 0.41 million in Wut were matched on the sequences of the annotated genes ${ }^{2}$ (Table 2). The half of them was located on antisense strands of the annotated genes (Fig. 6). They included 44 Huaq-specific small RNAs and 27 Wut-specific small RNAs. We set the threshold of 2-fold changes as standards of difference in abundance. As a result, 238 small RNAs in Huaq showed higher abundance than in Wut, while 77 small RNAs in Wut displayed higher abundance than in Huaq (Fig. 7A).

To detect the putative target genes of these small RNAs, the small RNAs were applied to match with their possible target genes of Arabidopsis and B. rapa. Potential targets (homologous to Arabidopsis genes) were displayed in Supplementary Table 2. The putative targets included hormone-related genes such as CTR1, AREB3 and ATCHX4 (Fig. 7B). The abundance of the small RNAs matched on CTR1, AREB3 genes were higher in Huaq, while that matched on ATCHX4 was higher in Wut. ATIPT3 regulates cytokinin biosynthesis. Small RNAs matched with ATIPT3 was more abundant in Huaq than in Wut. The small RNAs matched on ATCOAA, MEE39, and ATCAP-E1 were also more abundant in Huaq while those matched with ATCS-C (Wut-enriched), VHA-A, and ATNTH2 were more abundant in Wut than in Huaq. These results showed that many small RNAs that may target at the important genes were different in abundance between Huaq and Wut.

\section{Discussion}

Comparative profiling shows remarkable difference in types and abundance of small RNAs between different varieties of Chinese cabbage

The whole view of small RNAs from small RNA deep sequencing provides information of non-coding RNAs in Chinese cabbage. These small RNAs are derived from nuclear, chloroplast and mitochondria genomes. In Huaq seedlings, the total numbers of 21-24 nt small RNAs are more abundant than in Wut. High amount of small RNAs in Huaq plants is mainly because of more types of small RNAs (unique small RNAs). Meanwhile, the gene-derived small RNAs in Huaq plants are more than those of Wut. The genederived small RNAs are mainly composed of small RNAs generated on antisense strands of the annotated genes. In the other words, the natural antisense transcripts (NATs) in Huaq plants generate more nat-siRNAs than in Wut plants. nat-siRNA plays an important role in plant development, stress response and disease resistance at transcriptional and post-transcriptional levels (Liu et al., 2008; Swiezewski et al., 2007). More nat-siRNAs in Wut plants indicate that development of Wut plants are vulnerable to stress and disease. Among 78 miRNAs, 3 miRNAs were Wut-specific. 76.9\% miRNAs were expressed predominantly in Huaq seedlings, and $23.1 \%$ miRNAs predominant in Wut seedlings.

Chloroplasts are important for photosynthesis and respiration. Abundance comparison of csRNA and rRNA reveals that small RNAs are more abundant in Wut seedlings than in Huaq seedlings, consistent 
with dark-green leaves of Wut seedlings. Ribosome biogenesis is a complex process comprising transcription, modification, and processing of ribosomal RNA and production of ribosomal proteins (Deisenroth and Zhang, 2010). Moreover, abundant rRNA-derived chloroplast small RNAs in Wut seedlings may reflect strong activity of photosynthesis, a process in which small RNAs are involved.

Genome-wide survey of small RNAs provides a basis for study of molecular

mechanism governing leaf traits of Chinese cabbage

The difference in miRNA abundance between Huaq and Wut may cause variance of some important development processes such as adaxial/abxial polarity, cell division and auxin response. In Arabidopsis, several conserved miRNAs regulate shape, size, color and curvature of leaves. miR156 targets at 11 of 17 SPL genes (Axtell and Bowman, 2008). Overexpression of SPL3, SPL4 and SPL5 result in adult characters of leaf shape on juvenile leaves and thus promote vegetative phase change and floral transition. Overexpression of miR156 results in prolonged expression of juvenile characters due to extended period of juvenile leaf shape (Chuck et al., 2007). In Huaq seedlings, miR156 accumulated more than in Wut seedlings. Coincidently, Huaq plants maintains longer period of juvenile leaf shape than Wut plants. miR172 accumulates more in Wut seedlings than Huaq. In Arabidopsis, miR172 is antagonist of miR156 and promotes flowering by repressing TOE1 and TOE2 (Glazinska et al., 2009), while the maize Cg1 mutant that overexpresses miR156 has reduced miR172 levels (Chuck et al., 2007). Higher level of miR172 in Wut may affect leaf traits by repression of miR156. The miR165/166 is conserved among seed plants and negatively regulates accumulation of members of the transcription factors HD-ZIP III families involved in meristem function, vascular patterning and adaxial cell fate (Wenkel et al., 2007; Zhong and Ye, 2007). Accumulation of miR165/166 on the abaxial side of leaf primordia delimits the HDZIP III transcripts in adaxial side of leaf and affects the polarity of the leaf(Juarez et al., 2004; Kidner and Martienssen, 2004). Overexpression of miR165/166 will conduct abaxial character of leaves. Huaq seedlings generate more miR166 than Wut seedlings. This difference could be reflected with leaf curvature. In Wut seedlings, miR319 accumulated less than in Huaq seedlings. It is interesting to determine whether this difference is linked to the phenotype of leaf margins of Wut. In Arabidopsis, miR319-mediated targets regulate leaf morphogenesis (Palatnik et al., 2003).

The correlation between miRNA expression and leaf traits in Chinese cabbage indicate their possible relation. It remains unknown whether these miRNAs are causally related to the certain leaf traits of Chinese cabbage. The functions of these small RNAs should be identified with the loss-of-function mutants and the transgenic plants, especially in the backgrounds of Chinese cabbage. Biogenesis of small RNAs must be rigorously regulated, and many small RNAs are expressed in a spatiotemporally regulated manner or in response to environmental stimuli (Chen, 2009). Total numbers of unique small RNAs in Huaq were much more than those of Wut, whereas total numbers of chloroplast-specific small RNAs derived from chloroplast genome of Wut were more than those of Huaq. Possibly, the genes responsible for a subset of small siRNAs or miRNAs are different in sequences and function, and thus exhibit different ability to direct biogenesis of small RNAs. For the purpose to investigate miRNA- 
mediated leaf development in Chinese cabbage, an attempt has been made in our lab to verify the sequences and functions of the genes responsible for biogenesis of small RNAs to analyze the morphological consequences of several miRNAs differentially expressed in Huaq and Wut. These small RNAs are useful for understanding molecular mechanism of leaf development and stress resistance and for genetic improvement of major agronomic traits.

Small RNAs are useful for developing of small RNA makers for leaf traits desirable for high yield and quality

The change in sequences and abundance of small RNAs may affect leaf development by altering expression of targeted genes. Among Huaq- or Wut-specific small RNAs, there are a number of single nucleotide polymorphisms (SNPs). If some small RNAs are functional in leaf development, the SNPs may be linked with certain leaf traits. Then, the small RNAs with these SNPs may become the molecular makers for leaf shape, size, color or curvature. To find out small RNAs markers, we have started to establish the relationship between SNPs in small RNAs and some quantitative trait loci (QTLs) responsible for leaf shape, size, color or curvature of Chinese cabbage. On the other hand, abundance of some small RNAs may be linked with certain leaf traits. Huaq has stronger ability to generate classic small RNAs than Wut. These small RNAs included those not only generated from the region corresponding to the known small RNAs but also from the externally and internally transcribed regions and space regions. Small RNAs are considered to be sequence-specific, depending on nucleotides, such as DNA and histone methylation and posttranscriptional regulation at RNA levels (Chen, 2009). Functional small RNAs are capable of affecting leaf phenotypes. It is possible for us to disclose the linkage relationship between abundance of small RNAs and leaf traits. Some functional small RNAs could be used as expression markers for leaf traits of Chinese cabbage. The yield and quality of Chinese cabbage are highly dependent on leaf shape, size, color and curvature. The vegetable breeders usually design these leaf traits according to the consumer's requirement but seldom success in breeding of the desirable cultivars. One explanation for this difficulty is that the molecular makers for leaf shape, size, color and curvature are rare. The SNPs and abundance of small RNAs in Chinese cabbage should be a basis for exploring molecular markers of leaf variance.

\section{Declarations}

\section{Conflict of Interest Statement}

The authors declare that the research was conducted in the absence of any commercial or financial relationships that could be construed as a potential conflict of interest.

\section{Acknowledgements}

This work was supported by grants from National Program on Key Basic Research Projects (The National Basic Research Program (973): 2012CB113903). 


\section{Footnotes}

${ }^{1}$ http://brassicadb.org/brad/blastPage.php

${ }^{2}$ http://www.ncbi.nlm.nih.gov/geo/query/acc.cgi?token=vlannoewwkioadc\&acc=GSE

\section{References}

Adenot, X., T. Elmayan, D. Lauressergues, S. Boutet, N. Bouche, V. Gasciolli, and H. Vaucheret, 2006: DRB4dependent TAS3 trans-acting siRNAs control leaf morphology through AG07. Curr Biol 16, 927-32.

Axtell, M.J., and J.L. Bowman, 2008: Evolution of plant microRNAs and their targets. Trends Plant Sci 13, 343-9.

Chen, X., 2009: Small RNAs and their roles in plant development. Annu Rev Cell Dev Biol 25, 21-44.

Chitwood, D.H., F.T. Nogueira, M.D. Howell, T.A. Montgomery, J.C. Carrington, and M.C. Timmermans, 2009: Pattern formation via small RNA mobility. Genes Dev 23, 549-54.

Chuck, G., A.M. Cigan, K. Saeteurn, and S. Hake, 2007: The heterochronic maize mutant Corngrass1 results from overexpression of a tandem microRNA. Nat Genet 39, 544-9.

Deisenroth, C., and Y. Zhang, 2010: Ribosome biogenesis surveillance: probing the ribosomal proteinMdm2-p53 pathway. Oncogene 29, 4253-60.

Fujii, H., T.J. Chiou, S.I. Lin, K. Aung, and J.K. Zhu, 2005: A miRNA involved in phosphate-starvation response in Arabidopsis. Curr Biol 15, 2038-43.

Glazinska, P., A. Zienkiewicz, W. Wojciechowski, and J. Kopcewicz, 2009: The putative miR172 target gene InAPETALA2-like is involved in the photoperiodic flower induction of Ipomoea nil. J Plant Physiol 166, 1801-13.

Hollister, J.D., L.M. Smith, Y.L. Guo, F. Ott, D. Weigel, and B.S. Gaut, 2011: Transposable elements and small RNAs contribute to gene expression divergence between Arabidopsis thaliana and Arabidopsis lyrata. Proc Natl Acad Sci U S A 108, 2322-7.

Jamalkandi, S.A., and A. Masoudi-Nejad, 2009: Reconstruction of Arabidopsis thaliana fully integrated small RNA pathway. Funct Integr Genomics 9, 419-32.

Juarez, M.T., J.S. Kui, J. Thomas, B.A. Heller, and M.C. Timmermans, 2004: microRNA-mediated repression of rolled leaf1 specifies maize leaf polarity. Nature $\mathbf{4 2 8}$, 84-8.

Kidner, C.A., and R.A. Martienssen, 2004: Spatially restricted microRNA directs leaf polarity through ARGONAUTE1. Nature 428, 81-4. 
Li, R., C. Yu, Y. Li, T.W. Lam, S.M. Yiu, K. Kristiansen, and J. Wang, 2009: SOAP2: an improved ultrafast tool for short read alignment. Bioinformatics 25, 1966-7.

Liu, H.H., X. Tian, Y.J. Li, C.A. Wu, and C.C. Zheng, 2008: Microarray-based analysis of stress-regulated microRNAs in Arabidopsis thaliana. RNA 14, 836-43.

Liu, Z., L. Jia, Y. Mao, and Y. He, 2010: Classification and quantification of leaf curvature. J Exp Bot 61, 2757-67.

Mallory, A.C., D.P. Bartel, and B. Bartel, 2005: MicroRNA-directed regulation of Arabidopsis AUXIN RESPONSE FACTOR17 is essential for proper development and modulates expression of early auxin response genes. Plant Cell 17, 1360-75.

Montgomery, T.A., M.D. Howell, J.T. Cuperus, D. Li, J.E. Hansen, A.L. Alexander, E.J. Chapman, N. Fahlgren, E. Allen, and J.C. Carrington, 2008: Specificity of ARGONAUTE7-miR390 interaction and dual functionality in TAS3 trans-acting siRNA formation. Cell 133, 128-41.

Mun, J.H., S.J. Kwon, T.J. Yang, Y.J. Seol, M. Jin, J.A. Kim, M.H. Lim, J.S. Kim, S. Baek, B.S. Choi, H.J. Yu, D.S. Kim, N. Kim, K.B. Lim, S.I. Lee, J.H. Hahn, Y.P. Lim, I. Bancroft, and B.S. Park, 2009: Genome-wide comparative analysis of the Brassica rapa gene space reveals genome shrinkage and differential loss of duplicated genes after whole genome triplication. Genome Biol 10, R111.

Nagasaki, H., J. Itoh, K. Hayashi, K. Hibara, N. Satoh-Nagasawa, M. Nosaka, M. Mukouhata, M. Ashikari, H. Kitano, M. Matsuoka, Y. Nagato, and Y. Sato, 2007: The small interfering RNA production pathway is required for shoot meristem initiation in rice. Proc Natl Acad Sci U S A 104, 14867-71.

Nikovics, K., T. Blein, A. Peaucelle, T. Ishida, H. Morin, M. Aida, and P. Laufs, 2006: The balance between the MIR164A and CUC2 genes controls leaf margin serration in Arabidopsis. Plant Cell 18, 2929-45.

Palatnik, J.F., E. Allen, X. Wu, C. Schommer, R. Schwab, J.C. Carrington, and D. Weigel, 2003: Control of leaf morphogenesis by microRNAs. Nature 425, 257-63.

Reyes, J.L., and N.H. Chua, 2007: ABA induction of miR159 controls transcript levels of two MYB factors during Arabidopsis seed germination. Plant J 49, 592-606.

Sieber, P., F. Wellmer, J. Gheyselinck, J.L. Riechmann, and E.M. Meyerowitz, 2007: Redundancy and specialization among plant microRNAs: role of the MIR164 family in developmental robustness. Development 134, 1051-60.

Steeves, T.A., and Sussex, I.M. , 1989: Patterns in plant development New York: Cambridge University Press.

Sunkar, R., A. Kapoor, and J.K. Zhu, 2006: Posttranscriptional induction of two Cu/Zn superoxide dismutase genes in Arabidopsis is mediated by downregulation of miR398 and important for oxidative 
stress tolerance. Plant Cell 18, 2051-65.

Swiezewski, S., P. Crevillen, F. Liu, J.R. Ecker, A. Jerzmanowski, and C. Dean, 2007: Small RNA-mediated chromatin silencing directed to the 3 ' region of the Arabidopsis gene encoding the developmental regulator, FLC. Proc Natl Acad Sci U S A 104, 3633-8.

Voinnet, 0., 2009: Origin, biogenesis, and activity of plant microRNAs. Cell 136, 669-87.

Wang, J.W., R. Schwab, B. Czech, E. Mica, and D. Weigel, 2008: Dual effects of miR156-targeted SPL genes and CYP78A5/KLUH on plastochron length and organ size in Arabidopsis thaliana. Plant Cell 20, 1231-43.

Wang, L., X. Yu, H. Wang, Y.Z. Lu, M. de Ruiter, M. Prins, and Y.K. He, 2011a: A novel class of heatresponsive small RNAs derived from the chloroplast genome of Chinese cabbage (Brassica rapa). BMC Genomics 12, 289.

Wang, X., H. Wang, J. Wang, R. Sun, J. Wu, S. Liu, Y. Bai, J.H. Mun, I. Bancroft, F. Cheng, S. Huang, X. Li, W. Hua, M. Freeling, J.C. Pires, A.H. Paterson, B. Chalhoub, B. Wang, A. Hayward, A.G. Sharpe, B.S. Park, B. Weisshaar, B. Liu, B. Li, C. Tong, C. Song, C. Duran, C. Peng, C. Geng, C. Koh, C. Lin, D. Edwards, D. Mu, D. Shen, E. Soumpourou, F. Li, F. Fraser, G. Conant, G. Lassalle, G.J. King, G. Bonnema, H. Tang, H. Belcram, H. Zhou, H. Hirakawa, H. Abe, H. Guo, H. Jin, I.A. Parkin, J. Batley, J.S. Kim, J. Just, J. Li, J. Xu, J. Deng, J.A. Kim, J. Yu, J. Meng, J. Min, J. Poulain, K. Hatakeyama, K. Wu, L. Wang, L. Fang, M. Trick, M.G. Links, M. Zhao, M. Jin, N. Ramchiary, N. Drou, P.J. Berkman, Q. Cai, Q. Huang, R. Li, S. Tabata, S. Cheng, S. Zhang, S. Sato, S. Sun, S.J. Kwon, S.R. Choi, T.H. Lee, W. Fan, X. Zhao, X. Tan, X. Xu, Y. Wang, Y. Qiu, Y. Yin, Y. Li, Y. Du, Y. Liao, Y. Lim, Y. Narusaka, Z. Wang, Z. Li, Z. Xiong, and Z. Zhang, 2011b: The genome of the mesopolyploid crop species Brassica rapa. Nat Genet 43, 1035-9.

Wenkel, S., J. Emery, B.H. Hou, M.M. Evans, and M.K. Barton, 2007: A feedback regulatory module formed by LITTLE ZIPPER and HD-ZIPIII genes. Plant Cell 19, 3379-90.

Wu, F., L. Yu, W. Cao, Y. Mao, Z. Liu, and Y. He, 2007: The N-terminal double-stranded RNA binding domains of Arabidopsis HYPONASTIC LEAVES1 are sufficient for pre-microRNA processing. Plant Cell 19, 914-25.

Wu, M.F., Q. Tian, and J.W. Reed, 2006: Arabidopsis microRNA167 controls patterns of ARF6 and ARF8 expression, and regulates both female and male reproduction. Development 133, 4211-8.

Yu, L., X. Yu, R. Shen, and Y. He, 2005: HYL1 gene maintains venation and polarity of leaves. Planta 221, 231-42.

Yu, X., H. Wang, Y. Lu, M. de Ruiter, M. Cariaso, M. Prins, A. van Tunen, and Y. He, 2011: Identification of conserved and novel microRNAs that are responsive to heat stress in Brassica rapa. J Exp Bot 63, 102538. 
Zhong, R., and Z.H. Ye, 2007: Regulation of HD-ZIP III Genes by MicroRNA 165. Plant Signal Behav 2, 3513.

\section{Figures}
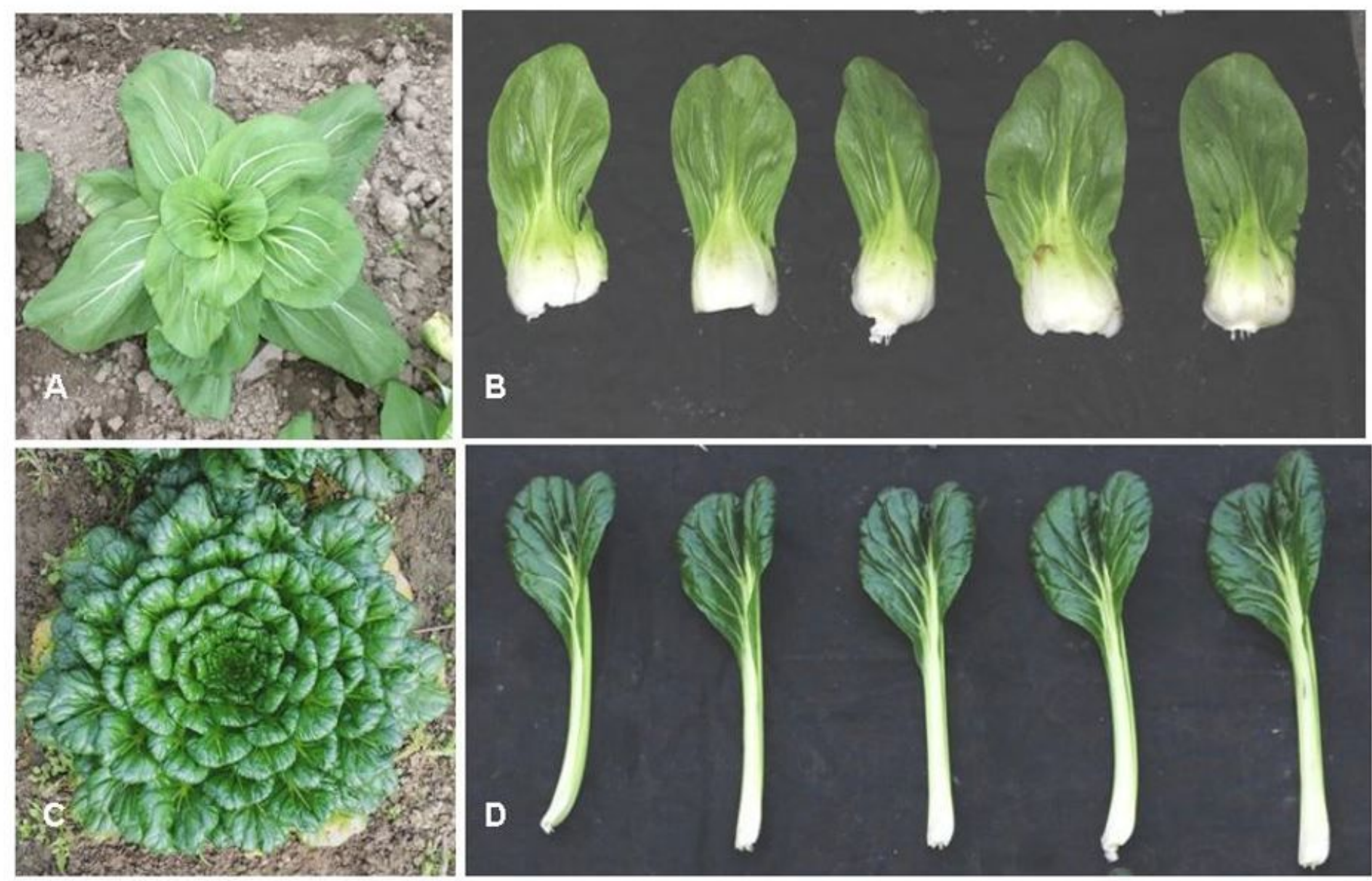

\section{Figure 1}

The seedlings of Huaq and Wut in the field. (A) Huaq seedling. (B) The leaves of Huaq seedling in order (from inside to outside). (C) Wut seedling. (D) The leaves of Wut seedling in order (from inside to outside). 
A
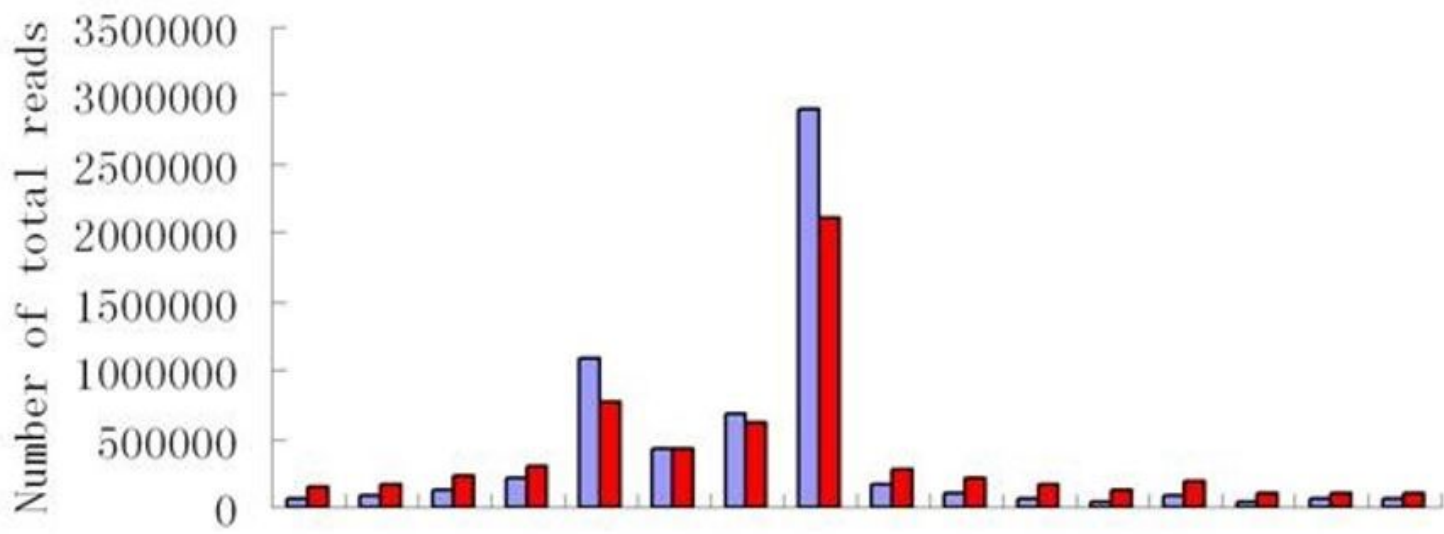

$\begin{array}{llllllllllllllll}17 & 18 & 19 & 20 & 21 & 22 & 23 & 24 & 25 & 26 & 27 & 28 & 29 & 30 & 31 & 32\end{array}$

Length

Length distribution of small RNA unique reads

B

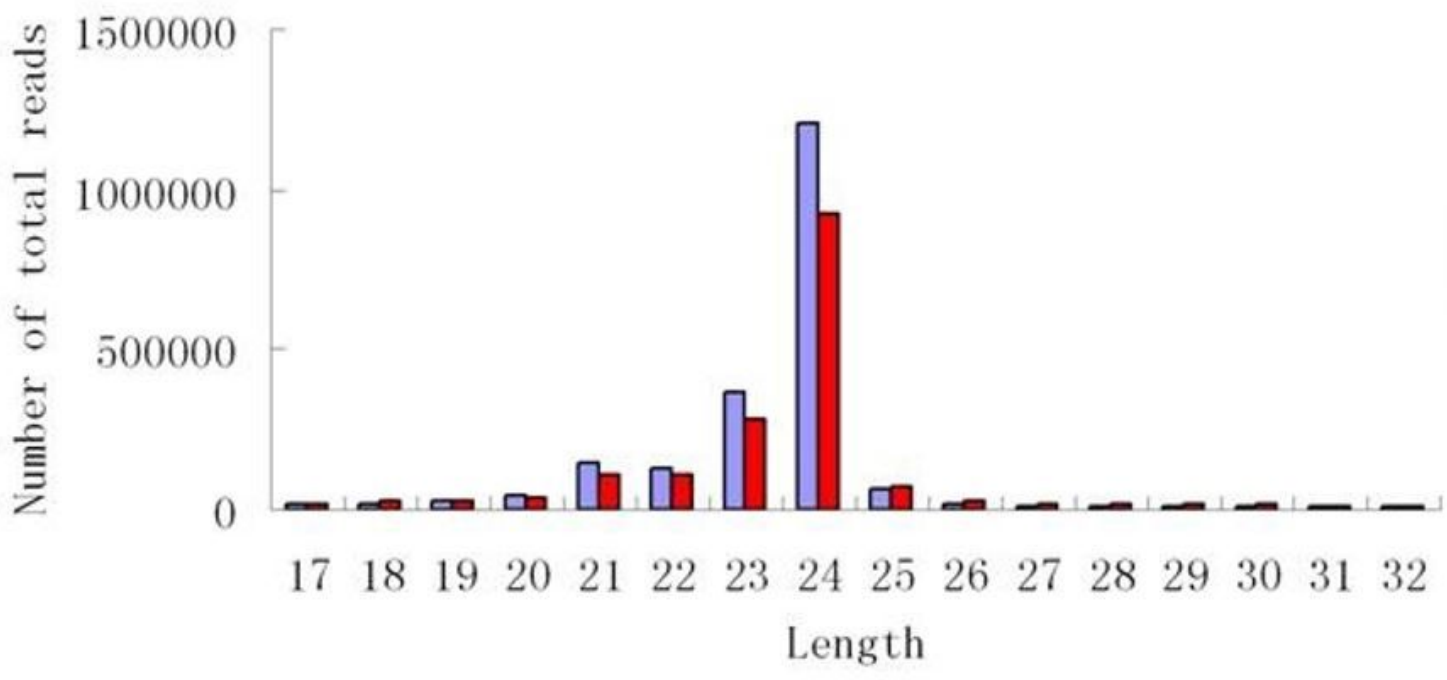

Figure 2

Length distribution of small RNAs in two databases of Huaq and Wut, (A) Length distribution of total reads in Huaq and Wut seedlings. (B) Length distribution of unique reads in Huaq and Wut seedlings. 
A Huaq unique reads annotation

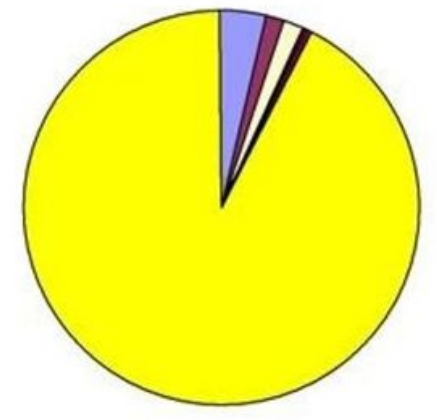

B
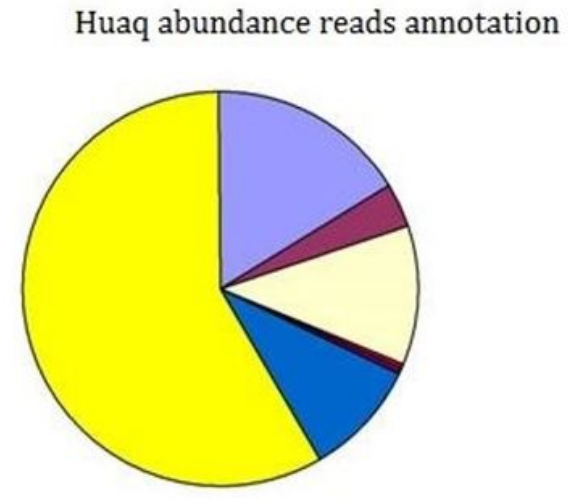

C

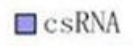

$\square$ mitRNA

$\square \mathrm{rRNA}$

atRA

- $\mathrm{TE}$

$\square$ snoRNA

amiRNA

口other

D

Wut abundance reads annotation

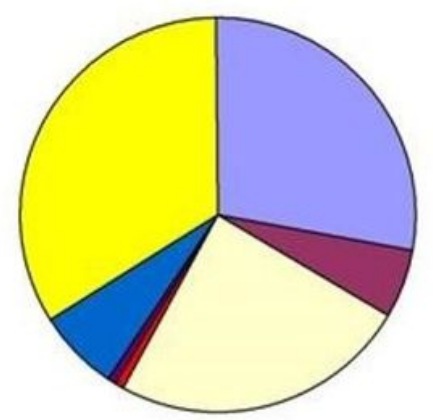

$\square \operatorname{csRNA}$

$\square$ mitRNA

$\square$ rRNA

$\square$ tRNA

口TE

$\square$ snoRNA

amiRNA

口other $\square \operatorname{csRNA}$

$\square$ mitRNA

$\square$ rRNA

口 tRNA

$\square \mathrm{TE}$

$\square$ snoRNA

$\square$ miRNA

$\square$ other

\section{Figure 3}

The classification of small RNAs. The small RNAs were classified into chloroplast-specific small RNAs (csRNA), mitochondria-specific small RNAs (mitRNA), ribosome RNA (rRNA), transfer RNA (tRNA), small RNAs derived from transposable elements (TE), small nucleolar RNA (snoRNA), miRNA and other RNA (other). (A) Proportions of classified unique small RNAs in Huaq seedlings. (B) The abundance distribution of classified small RNAs in Wut seedlings. (C) Proportions of classified unique small RNAs from total qualified small RNAs in Huaq seedlings. (D) The abundance distribution of classified small RNAs in Wut seedlings. 


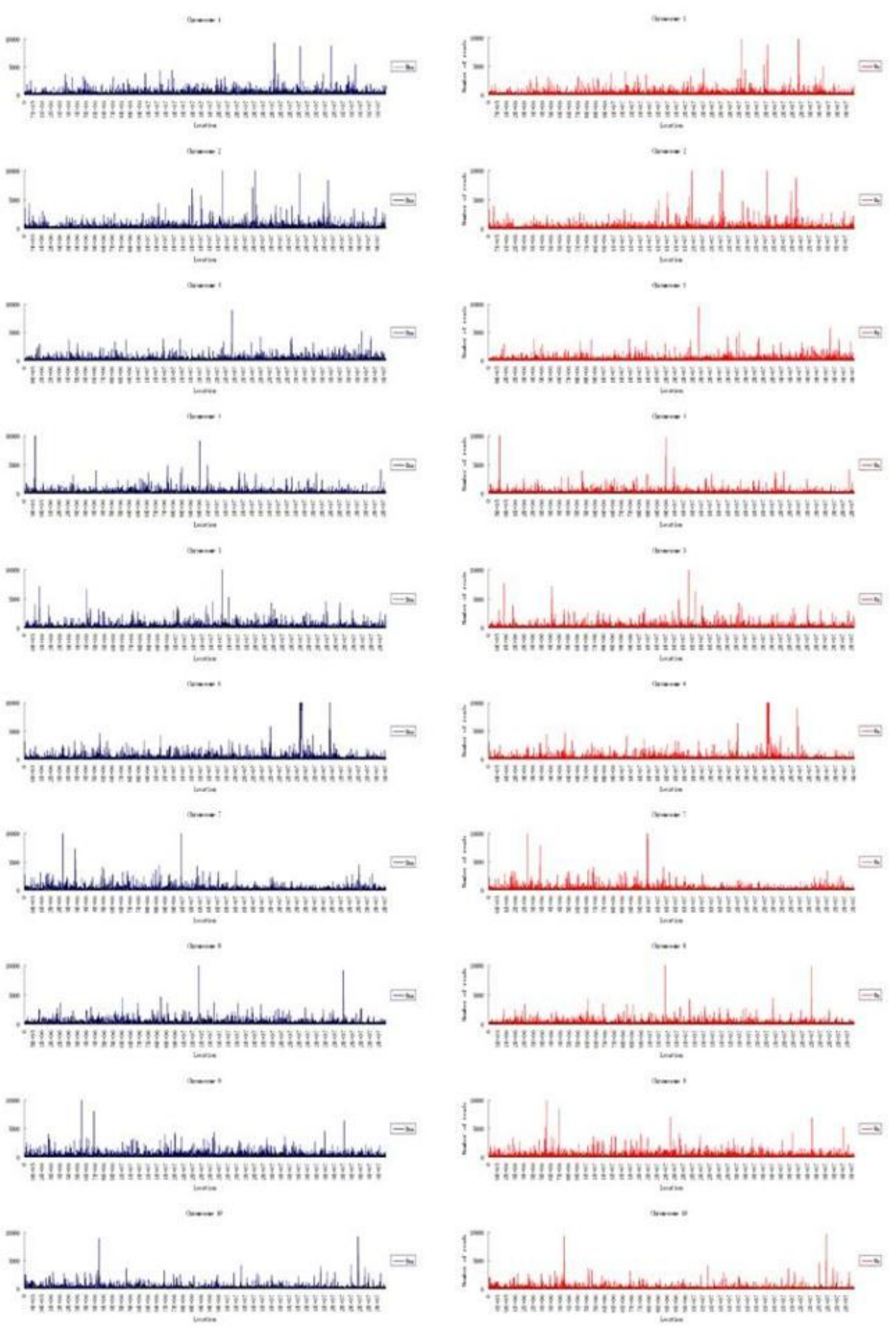

\section{Figure 4}

The distribution and abundance of small RNAs on the genome of B.rapa. A hot spot appears at the end of 9th chromosome in Wut but not in Huaq. 


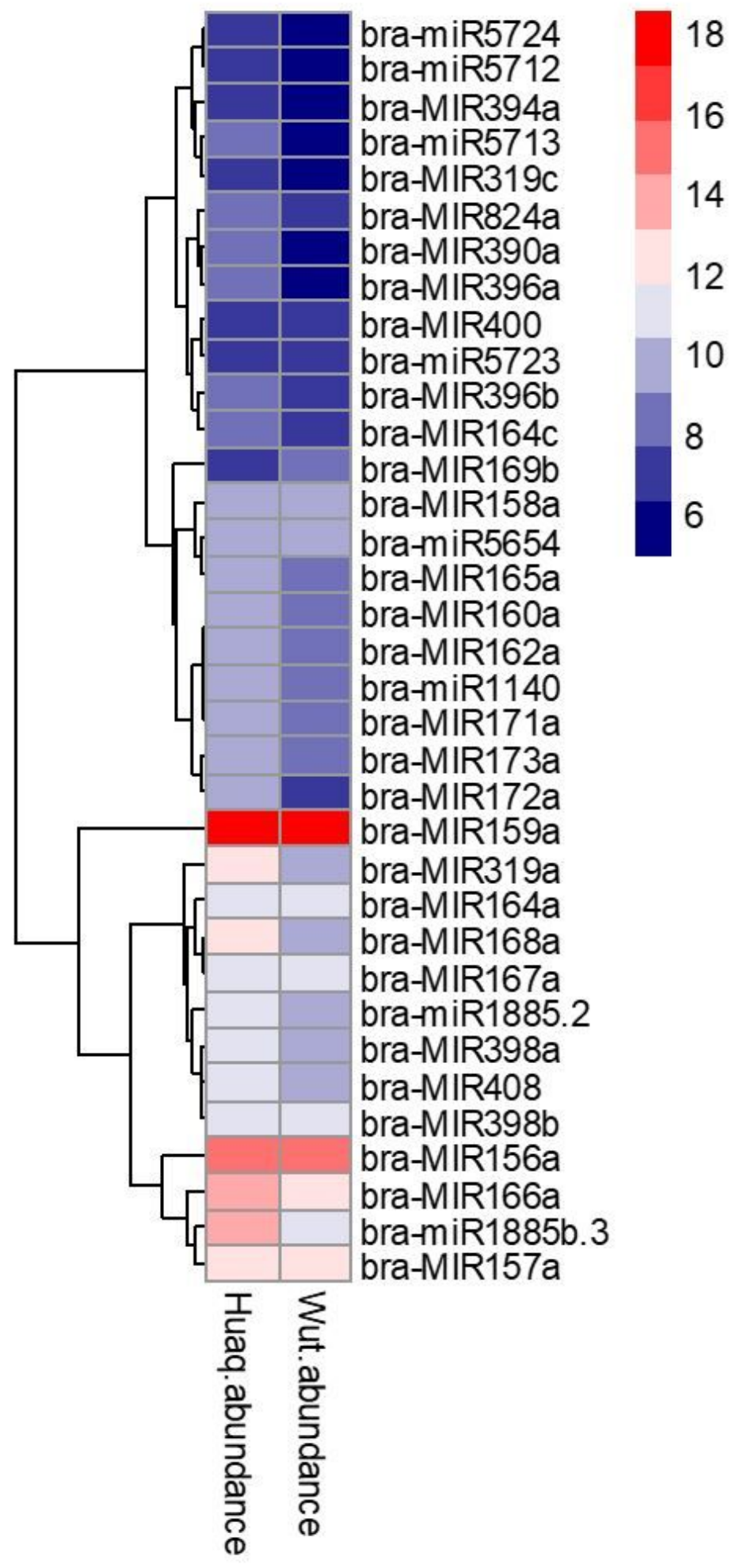

Figure 5

The abundance of the matched miRNAs, the heatmap of miRNAs of Wut and Huaq for noted miRNAs . 
Distribution of small RNA derived form sense transcripts

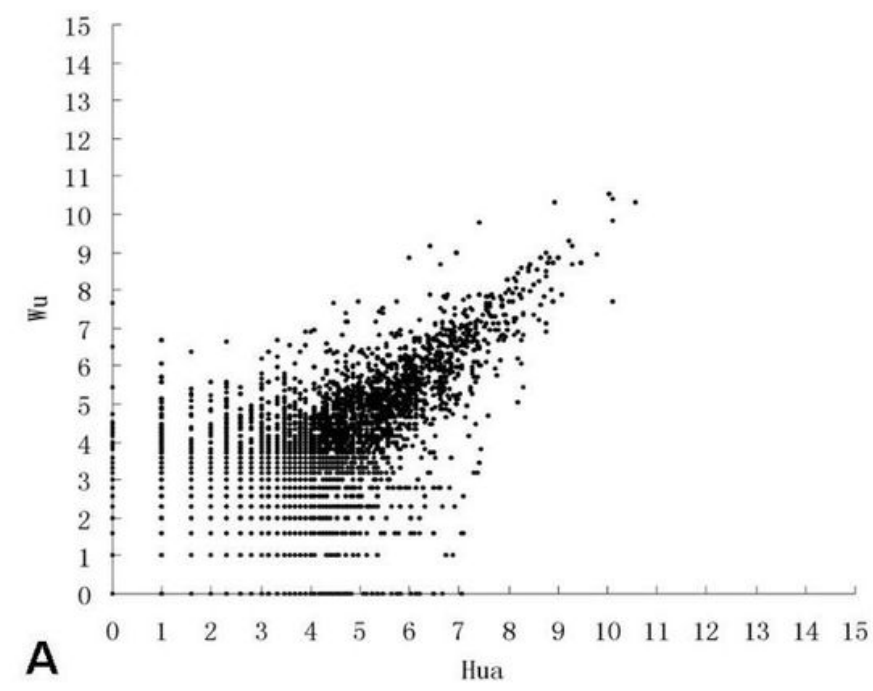

Distribution of small RNA derived from antisense transcripts

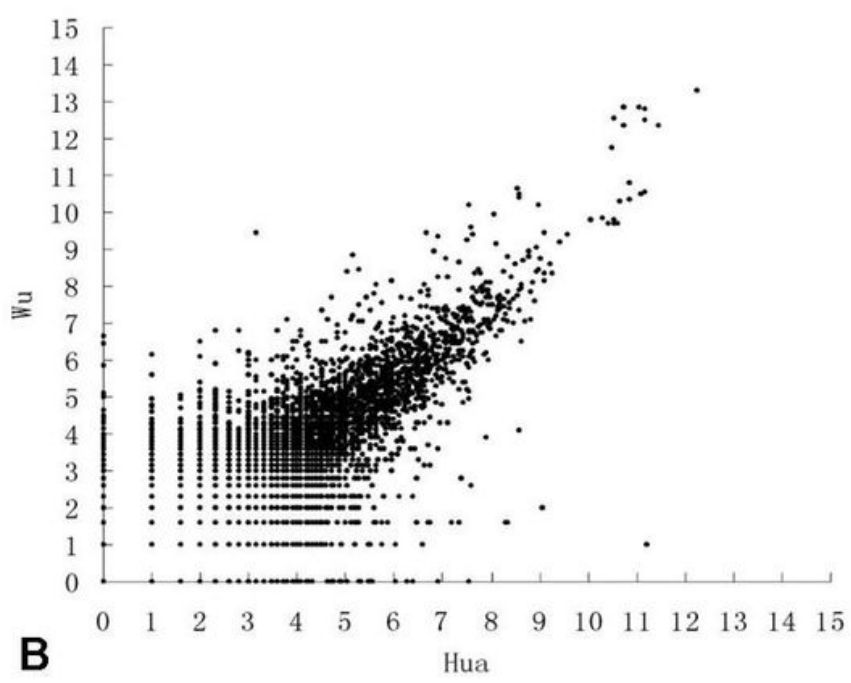

Figure 6

The distribution of small RNAs mapped to the annotated genes of Huaq and Wut. (A) The distribution of small RNAs derived from sense strands of the annotated genes. (B) The distribution of small RNAs derived from the antisense strands of the annotated genes. The draft was draw by logarithmic value of abundance. 
Small RNA located in antisense transcripts

A

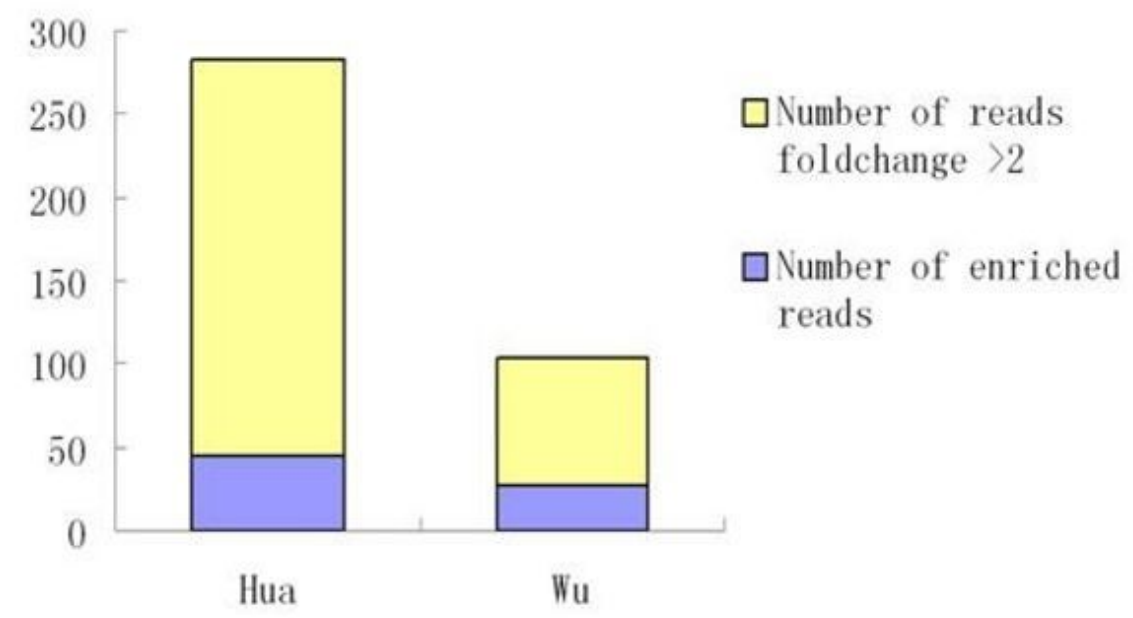

B

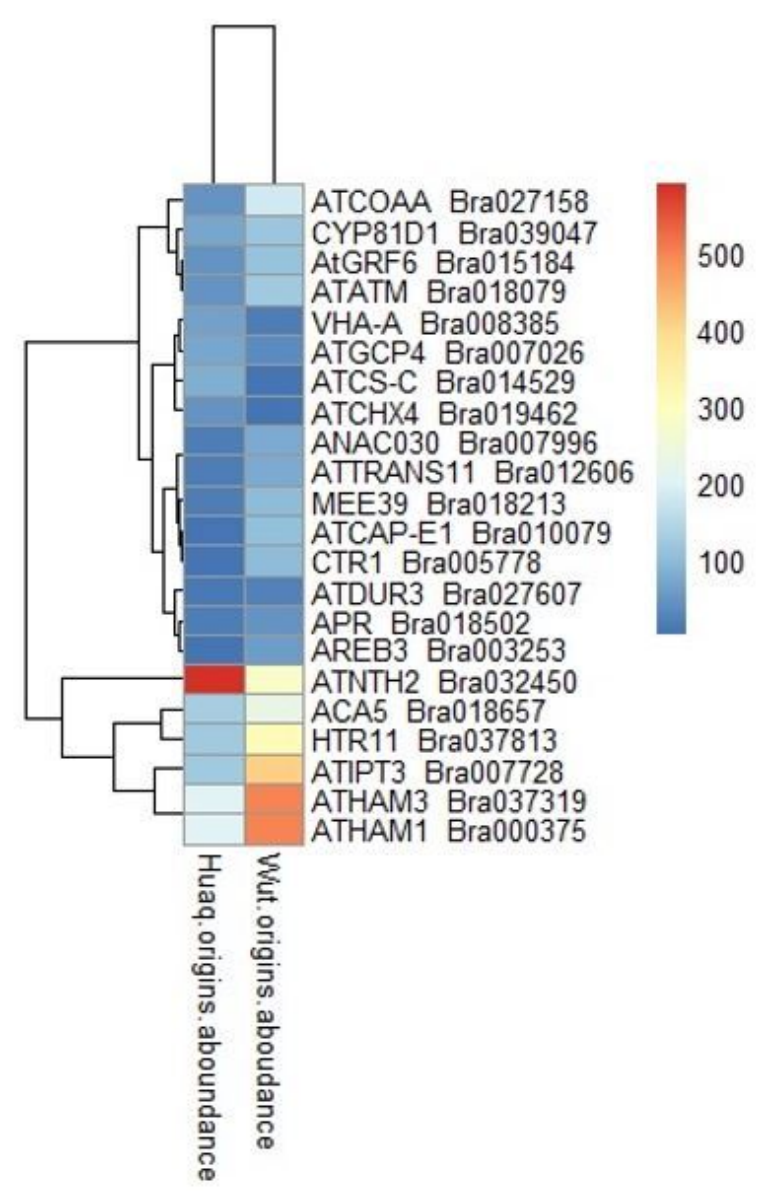

\section{Figure 7}

The abundance of small RNAs located in antisense strands of the annotated genes. (A) The number of the small RNAs located in the antisense strands. (B) The abundance of small RNAs located in antisense strands of Brassica genes (at the right), corresponding to orthologous genes of Arabidopsis (at the left). 